\title{
Connecting Bitcoin Blockchain with Digital Learning Chain Structure in Education
}

\section{Krisztián Bálintt ${ }^{1,2}$, Dragan Cvetković ${ }^{1}$, Márta Takács ${ }^{3}$, Ildikó Holik $^{4}$, Alex Tóth ${ }^{5}$}

${ }^{1}$ Singidunum University, Danijelova 32, 11000, Belgrade, Serbia

${ }^{2}$ Óbuda University, Doctoral School on Safety and Security Sciences, Népszínház u. 8, 1081 Budapest, Hungary

${ }^{3}$ Óbuda University, John von Neumann Faculty of Informatics, Bécsi út 96/b, 1034 Budapest, Hungary

${ }^{4}$ Óbuda University, Ágoston Trefort Centre for Engineering Education, Népszínház u. 8, 1081 Budapest, Hungary

${ }^{5}$ Municipal Museum of Subotica, Trg Sinagoge 3, 24000 Subotica, Serbia

E-mails: kristian.balint.12@singimail.rs, dcvetkovic@singidunum.ac.rs takacs.marta@nik.uni-obuda.hu, holik.ildiko@tmpk.uni-obuda.hu, muzejsubotica@mts.rs

Abstract: In the present paper, an extension of the Bitcoin (BTC) structure is introduced, with a proprietary and unique DLCC (Digital Learning Chain Structure) blockchain, through which an automated bursary payment system could be constituted, which has, until this day, never been implemented at universities. Thus, an alternative is offered to achieve a bursary payment system, operating in a fully automatic, periodically repeating manner, never requiring supervision. In the DLCC blockchain, specifically, in the Smart Contract, the terms of contract are kept, concerning the bursaries, terms agreed by both parties (students and universities alike). A great advantage of the DLCC system is that it is able to extract the required grades from the electronic grade book, thus supervising the students' progress throughout the semesters. Upon reaching the end of each semester, if the student qualifies, meeting the requirements, the system will transfer the required quantity of cryptocurrency from the university's account automatically to the student's account. 


\section{Introduction}

Bitcoin is an open source digital currency, introduced in 2009 by an unknown user. The term is also used for the open source software managing the currency, as well as for the created distributed network. Bitcoin does not depend on central issuing agencies or authorities since it is supported on the distributed database stored at peer-to-peer network nodes. Bitcoins can be safely stored in a wallet, on a computer or mobile device, as well as in a cloud-based provider. Since the system cannot be controlled from the outside, the essence of the transaction remains hidden, which is why it is criticized for its potential illegal usage. At the same time, there are more and more applications using solutions based on Bitcoin blockchains.

Blockchain technologies make tracking and managing digital identities, both secure and efficient, resulting in seamless sign-on and reduced fraud. Be it banking, healthcare, national security, citizenship documentation or online retailing, identity authentication and authorization is a process intricately woven into commerce and culture worldwide. Blockchain technology can be applied to identity applications in the following areas: Digital Identities, Passports, EResidency, Birth Certificates, Wedding Certificates, and Ids [1].

Blockchain technology can also be used in transportation. It provides support for tracking the goods with a blockchain-based solution. This way it is always possible to pinpoint the exact location of the goods anywhere in the world, en route between the manufacturer and user. [2].

Medical records are notoriously scattered and erroneous, with inconsistent data handling processes, i.e. hospitals and clinics, are often forced to work with incorrect or incomplete patient records. Healthcare projects such as MedRec are using the blockchain as a means of facilitating data sharing while providing authentication and maintaining confidentiality [3].

Over time, blockchain technology has found its way into various industry segments, too, including peer-to-peer taxi rides and other similar services. Arcade City is a blockchain-based ride sharing and car-for-hire application. Naturally, payment is organized via crypto currency [4].

Dubai has set sights on becoming the world's first blockchain-powered state. In 2016, representatives of 30 government departments formed a committee dedicated to investigating opportunities across health records, shipping, business registration and preventing the spread of conflict diamonds [5].

Traditional systems tend to be cumbersome, error-prone and rather slow. Intermediaries are often needed to mediate the process and resolve conflicts. As expected, this causes stress, and costs time, and money. In contrast, users find the blockchain cheaper, more transparent, and more effective. Unsurprisingly, an increasing number of financial services use this system to introduce innovations, 
such as smart bonds and smart contracts. The former automatically pays bondholders their coupons once certain preprogrammed terms are met. The latter are digital contracts that self-execute and self-maintain, again when terms are met [6].

The New York-based Bitcoin exchange is working on Project Highline, a method of using the blockchain to settle and clear financial transactions in $\mathrm{T}+10$ minutes rather than the customary $\mathrm{T}+3$ or $\mathrm{T}+2$ days [7].

Blockchain technology can be used in everyday life for gift cards and customer loyalty programs. The aim is to increase security, given that it stops the intermediaries by using unique BTC blockchain-based controls. By excluding the intermediaries, the gift cards make their way directly from the vendors to the customers without the customers ever having to give out personal information [8].

The DLCC (Digital Learning Chain Structure) system could also provide great help in education, as it could provide simplicity and transparency to a given system. The structure of Bitcoin has specific characteristics, whose possibilities have not been fully utilized in education so far, whereas, the system based on bursary payment could be realized with its help.

The aim of the research presented in this paper is to create a bursary payment system in higher education system, based on Bitcoin's structure, which could be utilized successfully by the institutions of higher education in the near future. The DLCC structure includes the following important elements, including:

- The generation of students' Smart Contracts based on BTC;

- The interconnection of electronic school registry (grade book) with the smart contracts;

- The examination of bursary terms by concentration of these systems;

- Online accessibility.

The development of the DLCC structure is complete, functioning and has been tested in practice.

Both the university, as well as the students must be open to introducing this system in practice. To this end the authors conducted an empirical research with the participation of 187 university students from Hungary and 102 students from Romania, so as to discover the students' opinions on Bitcoin, and whether they would be ready to accept a bursary system based on Bitcoin.

The paper first describes Bitcoin's basic elements, followed by the detailed description of the Digital Learning Chain Structure system in place. As mentioned briefly above, a case study is added to the research results, where the views of students from Hungary and Romania concerning Bitcoin are uncovered. 


\section{Bitcoin Basics}

Bitcoin is one of many types of virtual currencies based on an algorithm that creates a direct peer-to-peer transaction system [9] using the necessary concepts for that.

The Bitcoin protocol implements an address propagation mechanism to help peers discover other peers in the P2P network. Each Bitcoin peer maintains a list of addresses of other peers in the network and each address is given a timestamp which determines its freshness. Peers can request addresses from this list from each other using GETADDR messages and unsolicitedly advertise addresses known to them using ADDR messages. Whenever a Bitcoin node receives an ADDR message, it decides individually for each address in the message whether or not to forward it to its neighbours. It first checks if:

- the total number of addresses in the corresponding ADDR message does not exceed 10, and

- the attached timestamp is not older than 10 minutes.

If either of these two checks fails, the address is not forwarded; otherwise the address is scheduled for forwarding to two of the node's neighbours in case the address is reachable and to one neighbour only if it is non-reachable. An address is considered reachable by a node if the node has a network interface associated with the same address family. Otherwise, the address is marked as unreachable [10].

The Bitcoin blockchain consists of a hashchain of blocks: every block contains an ordered set of transactions and a hash of the preceding block (starting from the initial, the so-called "genesis" block). The key part is the Proof-of-Work (PoW) aspect of the hashchain: a Bitcoin block contains nonces that a Bitcoin miner (i.e., a node attempting to add a block to the chain) must set in such a way that the hash of the entire block is smaller than a known target, which is typically a very small number. In the early days of Bitcoin, the performance scalability of its probabilistic PoW-based blockchain was not a major issue. Even today, Bitcoin works with a consensus latency of about an hour (for the recommended 6-block transaction confirmation), and with up to seven transactions per second peak throughput (with smallest 200-250 byte transactions). On top of this, the Bitcoin network uses a lot of power, which, in 2014, was roughly estimated to be in the ballpark of 0.1-10 GW2.1.4 [11].

Users transfer coins (BTCs) to each other by issuing a transaction. A transaction is formed by digitally signing a hash of the transaction through which a BTC was acquired. Given that in Bitcoin there is one-to-one correspondence between signature public keys and addresses, a transaction taking place between two addresses $\mathrm{aS}$ and $\mathrm{aR}$ has the following form: $\tau(\mathrm{aS} \rightarrow \mathrm{aR})=$ (source, $\mathrm{B}, \mathrm{aR}$, SIGskaS (source, B, aR) \}. Here, SIGskaS is the signature using the private key skaS that corresponds to the public key associated with the aS, B is the amount of 
BTCs transferred, and source is a reference to the most recent transaction that aS acquired the B BTCs from. After their creation, Bitcoin transactions are released in the Bitcoin network. Once the validity of $\tau$ is confirmed, aR can subsequently use this transaction as a reference to spend the acquired BTCs. Consequently, Bitcoin transactions form a public record and any user can verify the authenticity of a BTC by checking the chain of signatures of the transactions in which the BTC was involved [12].

\section{Solution for Communication between Bitcoin and DLCC Blockchains}

Although it is true that Bitcoin has an open source system based on mathematical algorithms, on the other hand, it is almost an impossible and unaccomplishable task to make changes to the BTC blockchain. This is due to the fact that the transactions are continuously built on each other from the beginning of the system. Therefore, if a change in the blockchain had to be made, it would be necessary to retrace it to the first ever transaction and that is a serious task. Hence, the changes would be most expedient if made to another blockchain, which, in turn, would be connected to the existing and stable Bitcoin blockchain. The creation of a DLCC blockchain would provide a new solution, which could be harnessed in the educational system. As a first step, this new blockchain would allow the signing of Smart Contracts. So far, the Smart Contracts have been exclusive elements of the Ethereum system. Created in 2015, they started to be widely used in 2016. The Smart Contract system has seen steady adoption, supporting tens of thousands of contracts, holding millions of dollars' worth of virtual coins [13]. Therefore, the base of DLCC would consist of Smart Contracts. Based on the experiences, the following advantages and drawbacks of Smart Contracts can be identified:

- Fair exchange between mutually distrustful parties with rich contract rules expressible in a programmable logic; this feature prevents parties from cheating by aborting an exchange protocol, yet removes the need for physical meetings and (potentially cheating) third-party intermediaries;

- Minimized interaction between parties also for a rich set of contracts expressible in a programmable logic, reducing opportunities for unwanted monitoring and tracking;

- $\quad$ Enriched transactions with external state by allowing input authenticated data feeds (attestations) provided by brokers on physical and other events outside the Smart Contract system, e.g., stock tickers, weather reports, etc. 
Unfortunately, despite all their benefits, these properties also have a dark side, potentially facilitating crime because:

- Fair exchange enables transactions between mutually distrustful criminal parties, eliminating the need for today's fragile reputation systems and/or potentially duplicitous or law-enforcement-infiltrated third-party intermediaries;

- Minimized interaction renders illegal activities harder for law enforcement to monitor [14].

Considering the recent experiences shared in the trade periodicals, which discuss the Smart Contracts, it would be possible to eliminate numerous drawbacks of DLCC, starting from the initial phase. Considering the nature of faculty systems, it is to be assumed that they are not invented and created for conducting criminal activities, but rather for raising the standards of educational institutions', as well as the students' level of satisfaction. For the accomplishment of the mentioned project, the School Boards must strive to wholeheartedly provide all competency, and maximum correctness.

\subsection{Soft-Fork and Hard-Fork in Bitcoin Terminology}

Connecting the Bitcoins blockchain with the DLCC blockchain is a challenging task. The accomplishment of communication between the two blockchains requires the involvement of Bitcoins protocol. There are two possibilities for this: the Soft-Fork and the Hard-Fork.

In Bitcoin, the terms Soft-Fork and Hard-Fork describe compatibility breaking changes in the Bitcoin protocol: should the community be irreconcilably divided on such an issue, the old version and the new version of Bitcoin could emerge as distinct projects thereafter. While both versions of the Bitcoin protocol are in use, the differences in acceptance may cause a lasting blockchain-fork, i.e. the two distinctly longest chains which are both considered valid by part of the network.

- $\quad$ Soft-Forks are forward compatible,

- Hard-Forks are not forward compatible [15].

A Hard-Fork occurs when blocks that would have previously been considered invalid, are now valid. Any Bitcoin user, miner, exchanger, etc. with the intention to stay in consensus with the network must upgrade their software during a HardFork; otherwise, some new block that the network accepts will appear as invalid to it.

A Soft-Fork occurs when blocks that would have previously been considered valid, are now invalid. Upgrading software during a consensus Soft-Fork is always optional for any Bitcoin user, miner or exchanger, with the following caveats: 
- If the Soft-Fork introduces a new feature that the user wishes to implement as either the sender or recipient, they must upgrade in order to use it.

- $\quad$ At least $51 \%$ of miners must upgrade to adopt the Soft-Fork, otherwise, it will always appear as the shortest chain and become orphaned by the network.

- Refusal to accept the Soft-Fork can reduce one's security. Given that Soft-Forked transactions are normally considered invalid, Bitcoin developers use various tricks to make these transactions appear valid, while also reducing the client's capacity to process exactly why they are valid [16]. The first figure presents the operation of the Soft-Fork blocks (Figure 1).

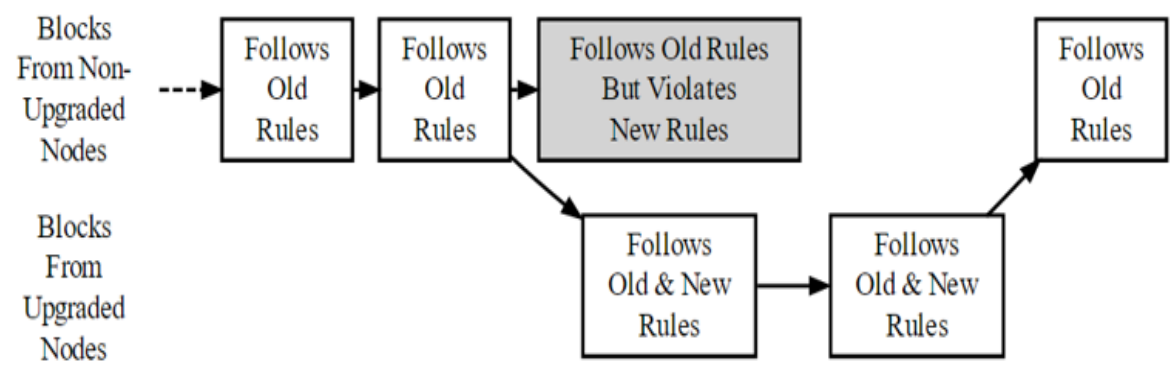

Figure 1

A Soft-Fork: Blocks violating new rules are made stale by the upgraded mining majority

For the implementation of the protocol between the BTC blockchain and DLCC blockchain, in this case, the best solution would be the Soft-Fork. The Figure 2 shows how to connect the DLCC blockchain with the Bitcoin blockchain. 


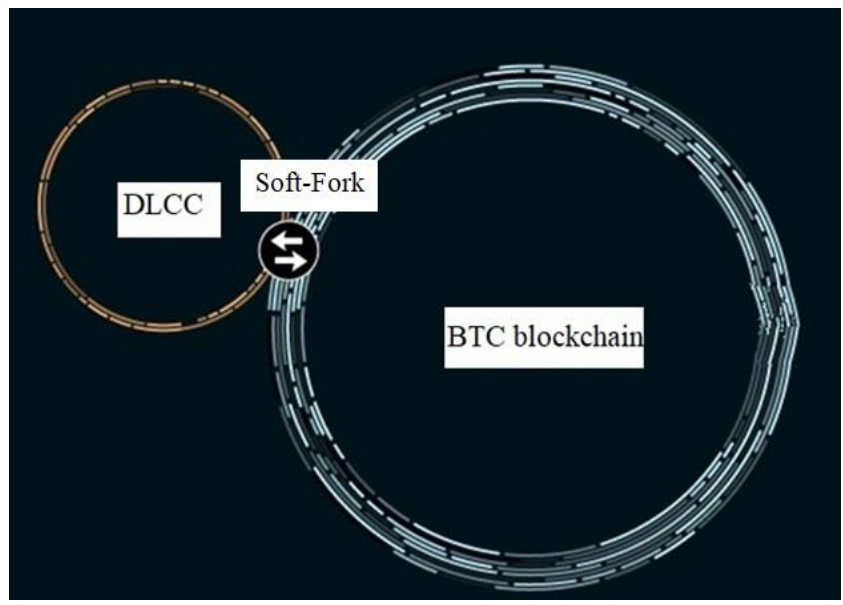

Figure 2

Link between DLCC's and Bitcoin's blockchains

The process of creating the DLCC blockchain can be seen in the Table 1 .

Table1

Blockchain creating process

Mychain-util generate DLCC

the default settings would be used:

/default mychain/DLCC/params.dat

params.dat include:

Contract addresses [receiver (student) IP address, sender (university) IP address], BTC wallet addresses [receiver (student) IP address, sender (university) IP address],

Terms of contract.

Next the DLCC blockchain would be initialized, and the genesis block would be mined

mychain DLCC

The server will be started in those few seconds after the genesis block has been found, then the node address needs to be connected:

DLCC@192.168.0.1:8008 
After these steps, the connection can be attempted from a second server:

mychain DLCC@192.168.0.1:8008

After the message confirming the chain has been initialized, the permission is not given for connection to the wallet. The address would be copied and pasted: 192.168.0.2

finally, permission for connection would be granted:

mychain DLCC grant 192.168.0.2 connect

At this point, an attempt can be made to reconnect to the second server, by a multi-chained DLCC - daemon. After the setup of the blockchain, the interactive mode would be used, where the setup would commence, so permissions addresses, peers, parameters of blockchain, native parameters, transaction meta-data, and streams would be given and mining would commence [17].

\section{Operating Principles of DLCC}

The application of networked computer systems has brought a great change in higher education in the form of electronic teaching materials, communication using web pages, and course administration by purposeful software systems [18].

The next step, utilizing these purposeful software systems, the upper management of the universities ought to perform management tasks, not yet used until now, in an automated way, with the help of DLCC. This is a novel possibility of performing these tasks, unavailable until several years ago. In order to create a successfully operating DLCC project, the adequate competence must be ensured not only from the university's side, but also from the students' side, as the digital literacy represents a mixture of consciousness, trending and capabilities, which enables the sufficient and safe application of digital means and institutions, with the aim of identifying, reaching, using, integrating, rating, and synthesizing of the digital sources, as well as the production of new knowledge and media publications, also with the goal of communication and mutual reflection regarding this process amongst ourselves [19].

The first step in the implementation of DLCC is signing the contract between the faculty and the student. This is to happen immediately after the students' enrollment to their first year of study. It would be highly useful in the case of enrollment for bursary. By signing the given contract, a possibility would arise for DLCC to automatically follow the students' results during their study years, with the help of marks in the electronic grade book. Thus, if a student meets the required terms, the bursary would be automatically be paid at the end of respective 
semesters. This solution would take off an enormous burden of faculties' administration. According to the contract, Krisztian is the university student, while Professor Dragan is the university representative, with whom Krisztián signs the bursary contract. The structure of DLCC Smart Contract consists of the elements presented in the Figure 3.

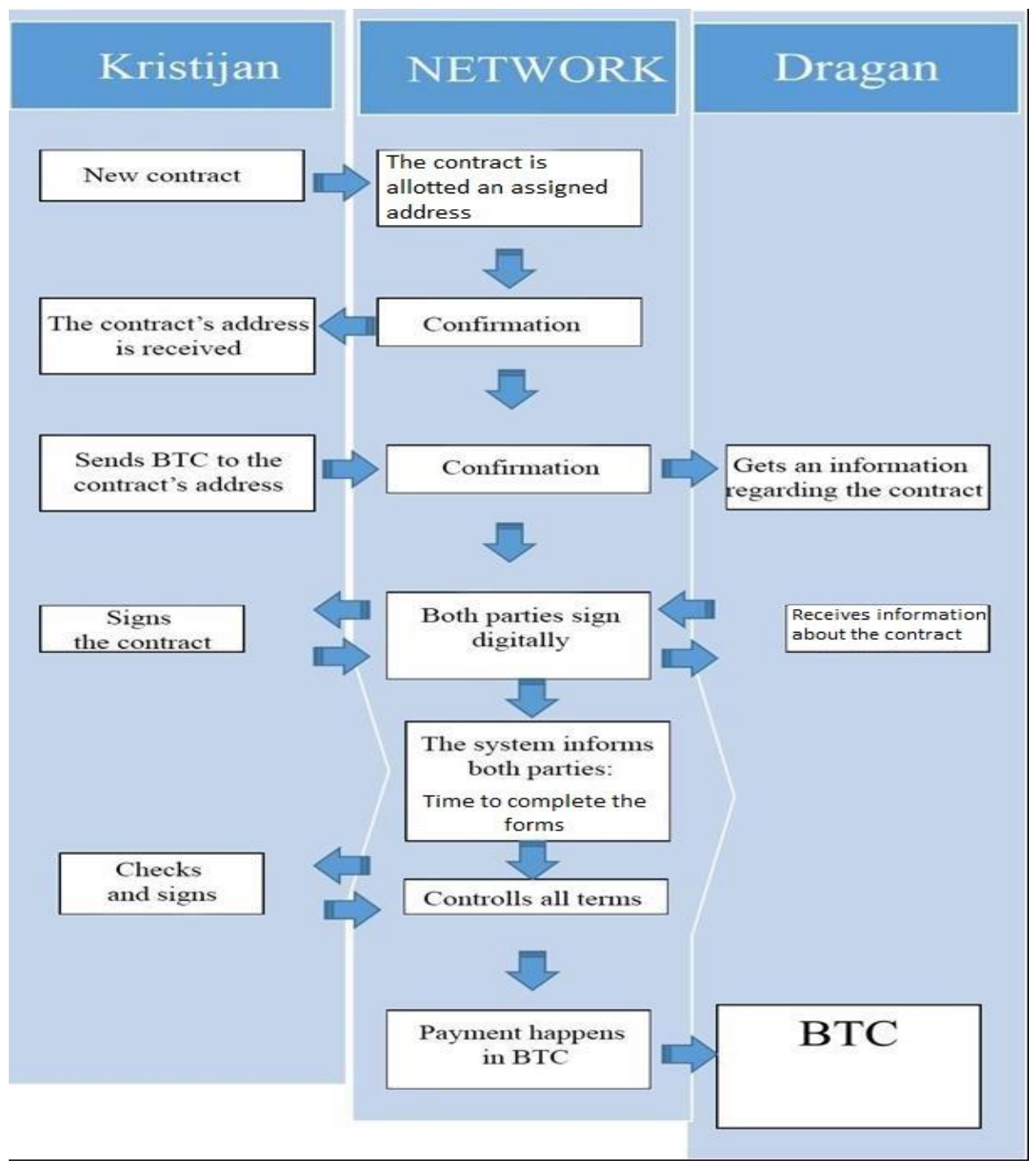

Figure 3

DLCC Bitcoin contract

Looking at this from another perspective, the mentioned innovation would be advantageous, because by the utilization of such systems, it would be possible to raise the students' financial proficiency in everyday life. This way, the students could be establishing a direct connection with modern financial systems even 
during their years of education. Such a solution is all the more thought-provoking given the fact that all people have finances, which need personal attention. Some people are prone to quick decision making, while others are prefer to obtain substantial quantity of information before each transaction, whereas there are also people who like to rely on their intuition. Therefore, aside from skills in economy, financial proficiency is considerably determined by societal and social relations, monetary attitudes and traits of personality. International research has proven the existence of significant differences among youth, considering the acquisition, interpretation and implementation of financial knowledge [20]. Information aggregation is one of the key issues in development of intelligent systems [21]. The Smart Contract automatically loads information needed for its operation from many sources, requiring no more than a safe access to the Internet. Necessary information includes, for instance, the daily Bitcoin exchange rate, the date and time, or the records and marks from the electronic grade book. As DLCC is based on Bitcoin's structure, thus, its secrecy is outstanding, as BTC uses military grade encryption, while the school based computer systems do not, and the Smart Contract is formulated on these. Security threats are closely related to the overall level of software security in computer systems [22]. Therefore, the school-based computer systems need to be upgraded, concerning the adequate level of protection.

The structure of DLCC would consists of the elements described in the following sections.

\subsection{The Definition of Terms of Contract}

A great advantage of Smart Contracts is that there is no possibility of later modification or manipulation. For this reason, it is important to ensure that the contracts are constructed with due diligence. In these contracts, concerning the applications for bursary, the students would accept the obligation, and state that they will fulfill the necessary terms, while the faculty would warrant regular and timely payment. In addition, the regularity of payments would be defined with exact dates, as the system would execute these payments automatically. For example, it would be ensured that payments would be executed on the first day of every month, or on the last day of every semester. Such payments have a great advantage, as they are not affected by holidays, weekends, because Bitcoin's network functions ceaselessly every day of the year.

\subsection{Electronic Grade Book}

It is important to secure, even on the institution level, a unified registry of curriculum, and a connecting informational system for leaders, which is necessary for the functioning of educational institutions, so they handle the data emerging from their functioning in a consistent way [23]. The electronic registry fulfills 
these terms completely, as the electronic rating (awarding of marks) is completed in this system, and similarly, the later recalling of marks by DLCC, according to the terms of contract.

\subsection{Gathering of Information}

Firstly, DLCC collects the necessary information from the electronic diaries, in a way that it continuously extracts the marks of a student, and at the end of each semester, the total marks of the subjects. Secondly, the system follows the corresponding dates, with the aim of keeping up with the dates of mentioned marks. DLCC can also read the actual date from the electronic grade book, if it is available, and if unavailable, the system can perform this task, with the help of access to the Internet.

Apart from collecting information, the constant following of Bitcoin's exchange rate is also very important, as the price of Bitcoin is rather dynamic, as this kind of currency has gone through many years of turbulent evolution in past years [24]. The high fluctuation of the exchange rate is characteristic it [25], however, the exchange rate of Bitcoin seems to undergo stabilization [26]. For this reason, it is vital for DLCC to use the Internet so that it continuously traces the fluctuation of exchange rate, as it can greatly influence the amount of payments.

\subsection{Examination of Terms in Contracts}

DLCC categorizes and evaluates the collected information. It examines the fulfillment of terms in contracts. If the terms are met, the next step will take effect: the payment. If the student fails to meet the requirements from the contract, then DLCC does not allow the completion of payment.

\subsection{Payment}

Lastly, the DLCC system pays the student; it transfers the necessary money to the corresponding electronic wallet. To this end, the student needs to possess a digital wallet. The users keep their wallets on their own machines, but in order to minimizing the risk of theft, they can demand the service of online wallets. Every wallet is based on a pair of keys - a public key and a confidential key, these two fulfill different tasks. The public key creates the address: it is basically a string of letters and numbers ranging from 27 to 34 characters. The secret key is used to validate the transactions. The addresses do not contain data about the user, but by signing of the public keys, the transaction can be retrieved. Although the transactions in Bitcoin can be retrieved based on the addresses, the owners of individual addresses remain unknown [27].

The construction of the DLCC structure is presented in the fourth figure in the series. As can be seen, the first step is the definition of the contract conditions, 
then the system can extract the data from the electronic log, compile them, and finally analyze them as to whether or not the necessary requirements for payment were met. As a final step, the bursary is transferred to the student. It is vital that the university has an up-to-date firewall, in this way it is possible to prevent any loss of grades in the electronic log.

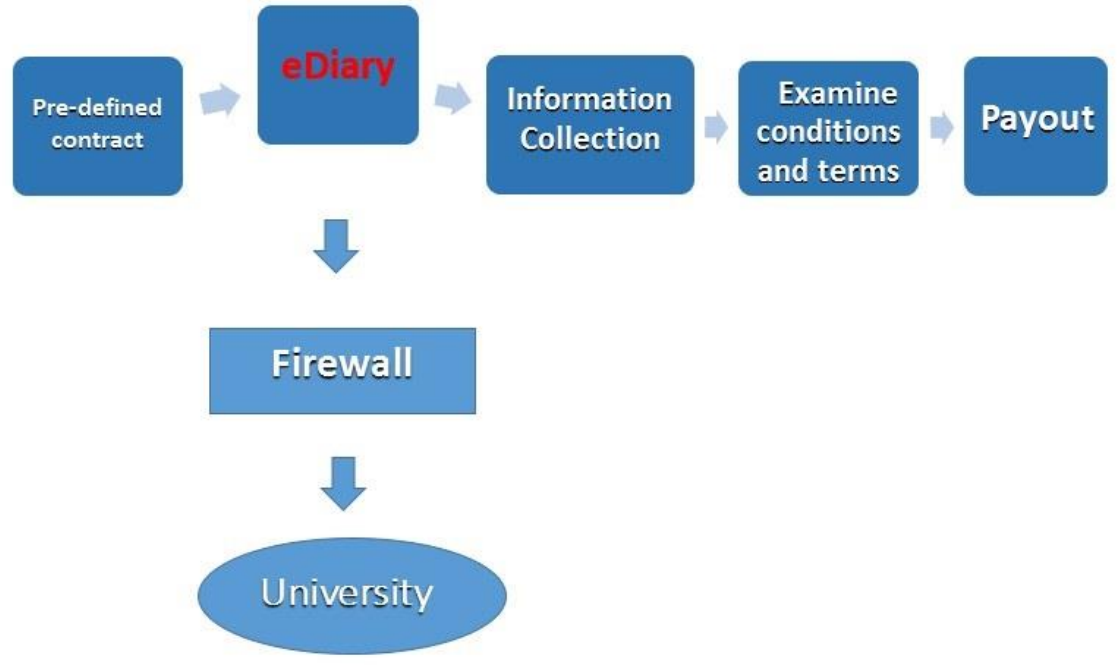

Figure 4

Main elements of the DLCC structure's operation

\section{Case Study about the Possible Introduction of the System for Students}

Before the commencement of the empiric investigation, it was an imperative to examine the economic situation of digital currency regarding countries where the research subjects (students) live, as the standpoints of some countries are were significantly divided regarding the topic of cryptocurrency, thereby influencing the test subjects' answers.

\subsection{Status of Hungary in the World of Cryptocurrency}

In Hungary, the following legislative decrees define the notion of electronic money:

The notions of electronic money and electronic means of payment have been defined by Law No. CXII. Since 1996, until its amendment on October 1, 2009, stating the following: 
"5.2 Electronic money: an amount (worth) of money, stored in electronic currency, issued in turn of receiving hard cash or a sum from a balance, which is accepted by other parties than the issuer, in order of electronic payment."

"5.3 Electronic means of payment: means of payment represents a substitute for hard cash - as such, particularly a card for storing value, or computer memory which is used to store electronic money, and with which the client is able to directly perform operations of payment " [28].

To date (2017), the National Bank of Hungary (MNB) has not formally recognized Bitcoin as a currency. According to the National Bank of Hungary, some of the means suitable for payment (specially Bitcoin) are by far more risky, than the electronic payment solutions already well-known to users (e.g. the credit cards, electronic money, etc.), as they do not have a formal issuer, they do not fall under supervision of any state-owned authorities or national banks, and there are no rules concerning the accountability, warranty, or damage management in existence. The National Bank of Hungary calls upon consumers' attention to be very aware, to act with utmost caution, before the use of such means of payment, for example Bitcoin [29].

\subsection{Status of Romania in the World of Cryptocurrency}

Up to 2017, the National Bank of Romania (BNR) has failed to pass any the normative regulation regarding Bitcoin, in other words, it does not prosecute, nor does it support the use of cryptocurrency. Romania's economy is surprisingly open towards the cryptocurrency in comparison to the neighboring countries, as one can obtain cryptocurrency in exchange for domestic currency in numerous places in Romania. There are 3 Bitcoin ATM machines working in Bucharest, Romania, since 2014. Within a one-way ATM transaction, it is only possible to exchange Romanian Lei for Bitcoins.

Moreover, thanks to the mutual cooperation of the operator of terminals, named Zebra Pay and Bitcoin Romania there is a possibility of more than 800 terminals and 160 cities in Romania to buy Bitcoins in exchange for domestic money. The transactions through the exchange agency named Coin trader carry a $4 \%$ transfer fee. With this new, simplified process of transaction, though, the transaction requires much less time [30]. However, regrettably, BTCX change, one of the greatest exchange offices of Romania concerning cryptocurrency, suspended its services for an indefinite period in early December, 2014. There is a background story about a misunderstanding between the lead programmer and the manager of the website. According to the programmer's statement, he had not received his salary; hence he closed down the servers, making them effectively inaccessible. These news caused significant surprise within the BTC community, as during almost 8 months of the website's operation, there were BTC transactions worth of 2 million dollars completed [31]. 


\subsection{Results of Empirical Research}

The empirical study was conducted with the aim to find out the attitude of Romanian and Hungarian students towards Bitcoin and the possible bursary system based on Bitcoin blockchain technology.

Table 2

Number of students participating in the present research, per states

\begin{tabular}{|c|l|c|}
\hline \multirow{5}{*}{ States } & \multicolumn{1}{|c|}{ Faculties } & \multicolumn{1}{|c|}{$\begin{array}{c}\text { Number of } \\
\text { students }\end{array}$} \\
\hline \multirow{5}{*}{ Hungary } & $\begin{array}{l}\text { Óbuda University - Kandó Kálmán Faculty of Electrical } \\
\text { Engineering - Electrical Engineering specialization }\end{array}$ & 76 \\
\cline { 2 - 3 } & $\begin{array}{l}\text { Budapest University of Technology and Economics - Social } \\
\text { sciences - Manager of technology specialization }\end{array}$ & 69 \\
\cline { 2 - 3 } & $\begin{array}{l}\text { Óbuda University - Ágoston Trefort Centre for Engineering } \\
\text { Education - Professor engineer specialization }\end{array}$ & 42 \\
\hline \multirow{5}{*}{ Romania } & $\begin{array}{l}\text { Babes-Bolyai University - Faculty of Economics and } \\
\text { Management - Financial and banking specialization }\end{array}$ & 43 \\
\cline { 2 - 3 } & $\begin{array}{l}\text { Babes-Bolyai University - Faculty of Economics and } \\
\text { Management - Management specialization }\end{array}$ & 15 \\
\cline { 2 - 3 } & $\begin{array}{l}\text { Babes-Bolyai University - Faculty of Economics and } \\
\text { Management - Tourism and economy specialization }\end{array}$ & 28 \\
\cline { 2 - 3 } & $\begin{array}{l}\text { Partium Catholic Faculty - Social Sciences and Economics - } \\
\text { Manager of tourism specialization }\end{array}$ & 16 \\
\hline
\end{tabular}

The hypothesis was: is it assumed that the participating 187 Hungarian and 102 Romanian students would accept their bursaries to be paid in Bitcoin?

The overall number of participants was initially 289. As a first step, it was examined if the students had heard about Bitcoin at all. A total of $41 \%$ of the students surveyed (that was 118 students) had not heard about Bitcoin at all, therefore, their further answers were ignored. This was necessary because these subjects could presumably not provide any relevant answers in a topic they had never heard of before. This led to the fact that in the later stages of research, the remaining 171 enquires were taken to be $100 \%$ of the students. The following questions were directed towards mapping the students' opinion about Bitcoin, as a prerequisite of the successful implementation of DLCC.

For the question whether Bitcoin would represent the alternative of traditional money, the students gave the following answers: $85 \%$ of the subjects answered negatively, while $15 \%$ gave a positive answer. This is fully understandable, as the dominance of paper money has long been present in everyday life, for this purpose, it is natural that the people have been forced to put their faith into it.

Considering the question if Bitcoin could represent the alternative of "traditional" electronic means of payment (E-banking, postal order, PayPal, etc.) in everyday 
life, the answers were more divided, as $56 \%$ of the subjects has given a positive, while $44 \%$ of them gave a negative answer. The significance level of the chisquare test regarding this question was $\mathrm{p}=0,417$, so, it can be stated that there is no significant correlation present in this case.

The data indicates that the students are certainly divided when considering Bitcoin's future. This is supported by the question examining the participants' views on how the cryptocurrency would prevail in the next 5 years. The obtained answers are depicted on the Likert scale below.

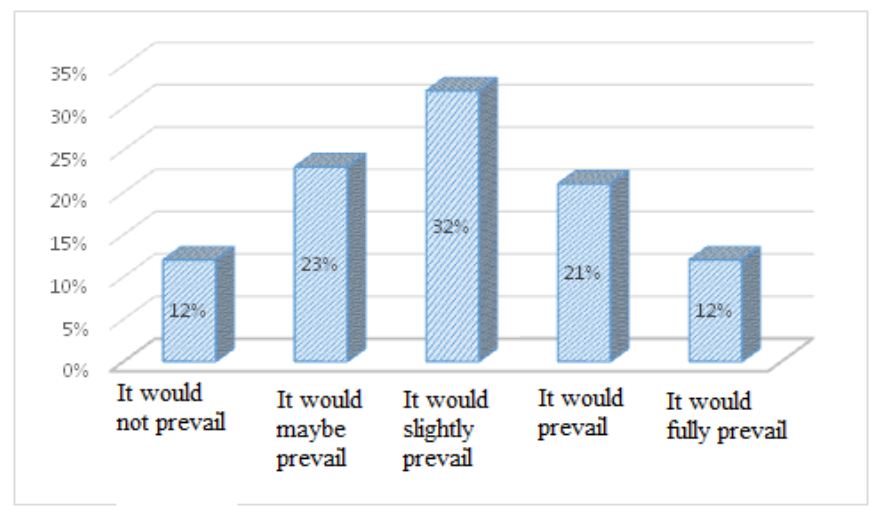

Figure5

What is your opinion about Bitcoin's future?

How will it prevail as a digital currency in the next five years? $(n=187)$

It can therefore be established that the opinions of students from various countries do not differ significantly regarding the question referring to how Bitcoin would prevail in the next five years (analysis of variance: $\mathrm{p}=0,619$ ).

The participating university students were asked to give their views on the following question "How would a certain fact influence Bitcoin's future, namely that Bitcoin does not belong under supervision of any state's jurisdiction, or central bank, instead, it is based on a mathematical structure? " The answers can be summarized as follows:

- Unfortunately, knowing Bitcoin's current status, I cannot answer this question (30\%).

- $\quad$ The systems used in banks are not fully dependable themselves (27\%).

- Regarding Bitcoin's future, in the long term, the P2P structure might have a negative influence (14\%).

- Looking at Bitcoin's future, in the long term, the P2P structure might have a positive influence (29\%).

Based on the answers given, it can be clearly seen that the future of Bitcoin remains obscure to the students, too. However, what is more surprising is that in 
their view, the systems used in banking are not fully dependable. The significance level of chi-square test is $p=0,614$, therefore, it can be stated that there is no significant correlation.

The last and also most important question was: "If you had the possibility of receiving a bursary, would you accept it in Bitcoins?" The percentage-ratio of positive and negative answers is shown in the graph below.

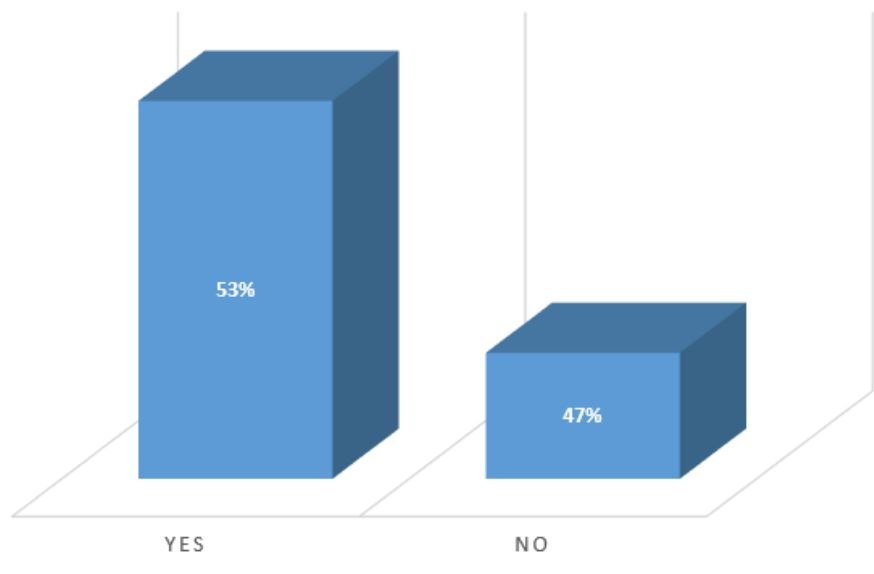

Figure 6

If you had the possibility of receiving a bursary, would you accept it in Bitcoins? $(n=187)$

It can be established that the hypothesis $\mathrm{n}$, according to which the students would accept a bursary paid in Bitcoins, has been prove, even if they felt both unsure and skeptic about the future of cryptocurrency.

\section{Conclusions}

The paper presents a system for introducing a Bitcoin-based bursary system. The technical realization, obtained results and a detailed description of the operational conditions are laid out in this work.

To sum up, in order for the DLCC system to work, there are a number of conditions that must be met in order to work. It is obvious that changing the Bitcoin blockchain is not an easy task, so the paper suggests the use of Soft-Fork for connecting two blockchains. In the DLCC system, within the smart contract's default settings (params.dat) the first step is to define the contract conditions, then setting up the address of the university, then the BTC wallet address of the student. The system extracts the study results from the electronic log and if those meet the requirements included in the contract, the bursary will be paid. Since the system is fully automatic after the setup, it is important to keep the firewall and virus protection up to date, so as to make sure that no data from the electronic log is compromised. Given that the DLCC system is based on the Bitcoin system, this enables a military-level protection that makes it almost impossible to manipulate data. 
Further, it is crucial that by the implementation of DLCC system, the administrative tasks would become simpler and more transparent. It is widely known that the institutions of higher education are usually struggling with meager financial bases; nevertheless, the realization of DLCC blockchain would not require enormous spending. One of the possible deficiencies of this system is the lack of trust, as it is utterly new and has never been utilized by the institutions of higher education until this day. If the top management of the universities gave the DLCC solution a vote of confidence, the realization of this Smart Contract bursary payment system could easily be accomplished. In the current situation, what is needed most, is a considerable amount of dedication, rather than serious financial investment.

\section{References}

[1] Ameer Rosic: 5 Blockchain Applications That Are Shaping Your Future, https://goo.gl/mY46eM (Download time: 2018-08-09) 2018

[2] Lányi, Márton.: Block technology for logistics. Bánki Reports, (Blokklánc technológia a logisztika szolgálatában), Bánki 2 Közlemények, 1.1 (2018): $5-10$

[3] Matteo Ginpietro Zago: 50+ Examples of How Blockchains are Taking Over the World, https://goo.gl/hyYr7y (Download time: 2018-08-09) 2018

[4] Jayanard Sagar: Arcade City Taps Blockchain Technology to Create NewAge Uber, https://goo.gl/AZUfZa, 2016 (Download time: 2018-08-09) 2018

[5] Bernard Mar: 35 Amazing Real World Examples of How Blockchain Is Changing Our World, https://goo.gl/Nv4yWx (Download time: 2018-0809) 2018

[6] Blockgeeks: 17 Blockchain Applications That Are Transforming Society, https://goo.gl/3qe1Fu (Download time: 2018-08-09) 2018

[7] Adam Hayes: Bitcoin 2.9 Applications, https://goo.gl/rauQa9 (Download time: 2018-08-09) 2018

[8] Upwork: 8 Blockchain Applications That Could Help Your Small Business, https://goo.gl/SVHNcq (Download time: 2018-08-09) 2018

[9] Wiseman, Scott A.: Property or Currency: The Tax Dilemma behind Bitcoin, Utah L. Rev. (2016): 417

[10] Biryukov, Alex, Dmitry Khovratovich, and Ivan Pustogarov: Deanonymisation of clients in Bitcoin P2P network, Proceedings of the 2014 ACM SIGSAC Conference on Computer and Communications Security. ACM, 2014 
[11] Vukolić, Marko: The quest for scalable blockchain fabric: Proof-of-work vs. BFT replication, International Workshop on Open Problems in Network Security. Springer, Cham, 2015

[12] Androulaki, Elli, et al: Evaluating user privacy in bitcoin, International Conference on Financial Cryptography and Data Security. Springer, Berlin, Heidelberg, 2013

[13] Miller, Mark S., and Marc Stiegler: The digital path: smart contracts and the Third World, 2003

[14] Juels, Ari, Ahmed Kosba, and Elaine Shi: The ring of gyges: Investigating the future of criminal smart contracts. Proceedings of the 2016 ACM SIGSAC, Conference on Computer and Communications Security. ACM, 2016

[15] StackExchange: Soft-Fork and Hard-Fork in Bitcoin terminology, https://goo.gl/TTsb3E (Download time: 2016-12-12)

[16] Blockchain Blog: A Brief History of Bitcoin Forks, https://goo.gl/YZJ3mV (Download time: 2016-12-12) 2016

[17] MultiChain: Getting Started with MultiChain, https://goo.gl/VO5tvC (Download time: 2016-12-12) 2016

[18] Horváth, László, and Imre J. Rudas: Course Modeling for Student Profile Based Flexible Higher Education on the Internet, J. UCS 12.9 (2006): 1254-1266

[19] Nyikes, Zoltán: Security awareness in the light of digital competence (A biztonságtudatosság a digitális kompetencia tükrében) 2016

[20] Andrea, Hornyák: Segmentation of young people on the basis of financial behavior (A fiatal korosztály szegmentálása a pénzügyi viselkedés alapján) Economists' Forum, Vol. 16, No. 112, 2013

[21] Rudas, Imre J., and János Fodor: Information aggregation in intelligent systems using generalized operators, International Journal of Computers Communications \& Control 1.1 (2006): 47-57

[22] Vokorokos, Liberios, Anton Baláž, and Branislav Madoš: Application security through sandbox virtualization, Acta Polytechnica Hungarica 12.1 (2015): 83-101

[23] Beke, Béla: Schedule of study records, (Tanulmányi nyilvántartás rendszerterve) https://goo.gl/AAL3da (Download time: 2016-12-12) (2009)

[24] Kristoufek, Ladislav: What are the main drivers of the Bitcoin price, Evidence from wavelet coherence analysis (2015): e0123923

[25] Rogojanu, Angela, and Liana Badea: The issue of competing currencies, 2014 
[26] Bonneau, Joseph, et al: SoK: Research perspectives and challenges for Bitcoin and cryptocurrencies, Security and Privacy (SP), 2015 IEEE Symposium on. IEEE, 2015

[27] Dion, Derek A: I'll Gladly Trade You Two Bits on Tuesday for a Byte Today: Bitcoin, Regulating Fraud in the E-Conomy of Hacker-Cash. U. Ill. JL Tech. \& Pol'y (2013): 165

[28] Gál, Veronika Alexandra, and Katalin Gáspár Bencéné Vér: E-money-local money (E-pénz-helyi pénz) Acta Scientiarum Socialium 15.38 (2013)

[29] Press release: National Bank of Hungary presumes virtual means of payment to be risky Bitcoin for example, https://goo.gl/0sfQJk (Download time: 2016-12-12) 2016

[30] Magyar Bitcoin Portál: It is possible to obtain Bitcoin in Romania at 874 terminals, (Romániában már 874 terminálon lehet bitcoint vásárolni), https://goo.gl/YXnyQi (Download time: 2016-12-12) 2016

[31] CCN: Bitcoin Exchange Closes after Lead Programmer Holds Servers Hostage, https://goo.gl/mHGw17 (Download time: 2016-12-12) 2016 